\title{
Probing the Element Distribution at the Organic-Inorganic Interface Using EDS
}

\author{
Meiken Falke ${ }^{1}$, Andi Kaeppel ${ }^{1}$, Baojun Yu ${ }^{2}$, Tobias Salge ${ }^{3}$ and Ralf Terborg ${ }^{1}$. \\ 1. Bruker Nano GmbH, Am Studio 2D, 12489, Berlin, Germany. \\ 2. Bruker Nano Analytics Division, Shanghai, China. \\ 3. Natural History Museum, Core Research Laboratories, Cromwell Road, London SW7 5BD, UK.
}

Based on analytical data, which probe the element distribution at the organic-inorganic interface, we suggest exploring respective options to complement light microscopy and other microscopy techniques with energy dispersive X-ray analysis (EDS) in the electron microscope to achieve element mapping.

Small particles of any kind embedded in organic material, be that toxic nanoparticles, calcifications, or markers down to the atomic level can be identified and analyzed by EDS. Even single atoms as light as $\mathrm{S}$ and $\mathrm{Si}$ on carbonaceous material are accessible by high-end techniques [1]. We show that the evaluation of the element distribution in combinations of soft and hard materials or light and heavy elements is routinely possible on a quantitative level in one single experiment, suitable preparation techniques provided. In situ element analysis in liquid or gas using EDS has been demonstrated as well [2]. However, the options to usefully complement light microscopy techniques established in life science with EDS need to be carefully investigated and chosen. We explain some of the possibilities, which could inspire new marking and immuno-labelling techniques using materials easily distinguishable by EDS or could just enable e.g. mapping the chemical composition of tissue, resin embedded cells (Fig.1) or of new porous biocompatible medical implant surfaces, decorated with nanoparticles to avoid infection [3].

The interface of soft and hard materials poses a challenge for EDS analysis. Samples resembling a close to natural state are often highly topographic, need analysis in liquid or gases or at low vacuum and can be beam sensitive. Preparation artefacts and absorption effects need to be considered. Thus, quantification models accounting for absorption effects are based on continuously improved atomic data bases and modelling. For electron transparent samples analyzed in TEM, the absolute Zeta-Factormethod proposed by $\mathrm{M}$. Watanabe has been implemented and tested on various materials additionally to the relative Cliff-Lorimer-method. Advancement in silicon drift detector (SDD) technology provides large single detectors, such as an oval detector (with up to $0.7 \mathrm{sr}$ solid angle) for atomic level analysis in TEM [1], multiple detector arrangements and an annular approach, such as the Flat Quad (with over 1sr solid collection angle) [4] suitable for high solid, high take-off angle experiments in SEM (Fig.2). Available standard and high end detector arrangements and respective successful measurement conditions and analysis strategies for life science used in STEM, SEM and T-SEM [5] will be explained, the latter including statistical analysis of nanoparticle distributions using TEM samples in SEM.

Figure 1 shows the investigation of the distribution of silver protein labels, osmium staining and light elements in a yeast cell using standard TEM EDS. The data were acquired in one single experiment and can be expressed quantitatively for each element identified. Figure 2 shows nanoparticle decorated sponge-like $\mathrm{TiO} 2$ which promotes cell proliferation on implant surfaces. The gold nanoparticles change the surface potential making it lethal for settling bacteria, an effect, also preserved in darkness [3].

In conclusion, EDS instrumentation and analysis techniques allow comprehensive qualitative and 
quantitative insights into the hard-soft materials interface. Suitable options to usefully combine EDS with other microscopy techniques in life science look promising and need to be thoroughly investigated.

[1] R M Stroud, et al., Appl. Phys. Lett. 108 (2016) p. 163101.

[2] E A Lewis et al., Chem Comm 50, No. 70 (2014) p. 10019-22.

[3] T Yang et al., Colloids and Surfaces B: Biointerfaces 145 (2016) pp. 597-606.

[4] R Terborg and M Rohde, Microsc. Microanal. 17 (2011) (Suppl.2) p. 892.

[5] V-D Hodoraba, et al., IOP Conf. Series: Mater. Sci. Eng. 109 (2016) p. 012006-1.

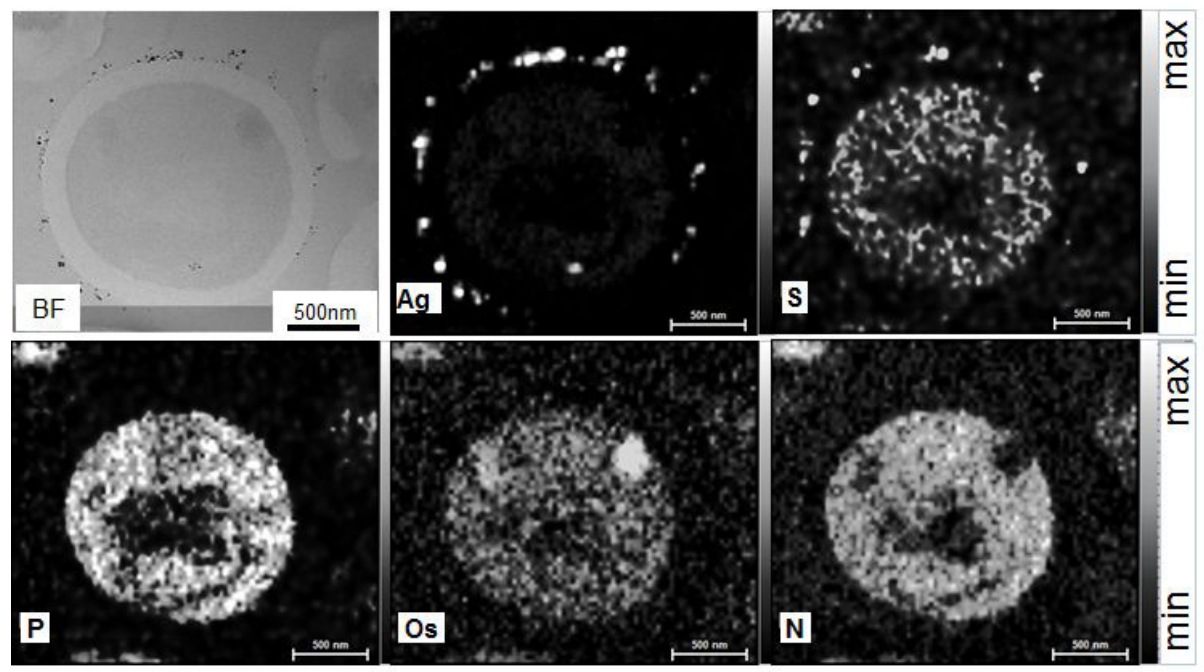

Figure 1. Resin embedded yeast cell with Ag labels for specific proteins. Element mapping reveals the distribution of many elements in one single experiment shown separately for clarity: light elements, such as $\mathrm{S}, \mathrm{N}$ and $\mathrm{P}$ and heavy elements, such as Ag label particles (dark in the bright field (BF) image, bright in the EDS Ag map) and the Os fixation. Acquisition using: 0.1sr standard EDS, conventional STEM at 300kV. Data courtesy: C. Slomianny, C. Biot Laboratory of Cell Physiology, Lille University, France.
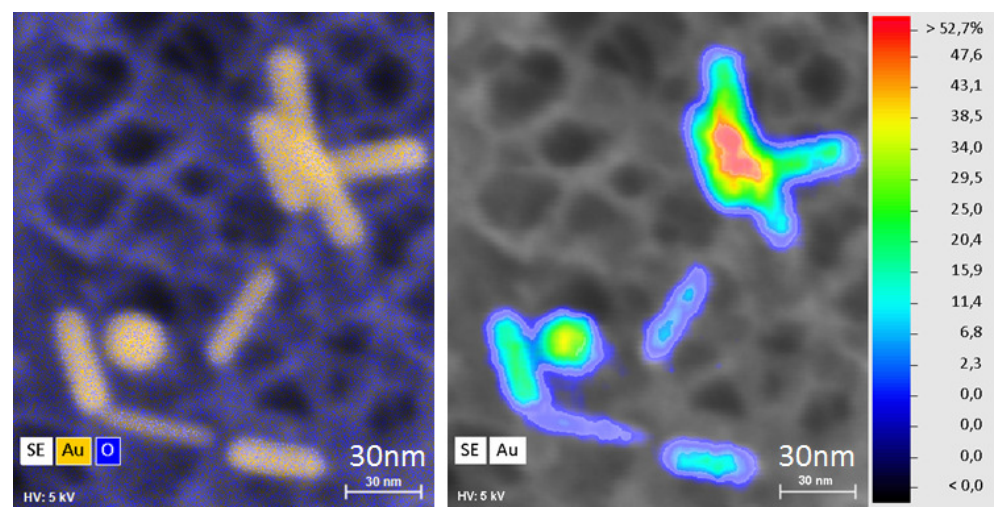

Figure 2. Au nanoparticle decorated porous $\mathrm{TiO} 2$ for implant surfaces. Left: $\mathrm{Au}$ and $\mathrm{O}$ net counts superimposed onto the SE image. Right: Au net count intensity normalized to maximum. Acquisition using: FEG SEM, annular detector with solid angle $\sim 1 \mathrm{sr}$ at $5 \mathrm{kV}$, input count rate $38 \mathrm{kcps}$. The high collection, high take-off angle low $\mathrm{kV}$ EDS in SEM helps to quickly judge the distribution and embedding of various nm sized particle types, sizes and shapes. Sample courtesy: Guangxi University and X. Liu and Y. Zeng, Shanghai Institute of Ceramics, Chinese Academy of Science, China. 